\title{
The regulatory mechanism of p38/MAPK in the chondrogenic differentiation from bone marrow mesenchymal stem cells
}

\author{
Ning Ma ${ }^{1}$, Xiao Teng ${ }^{1}$, Qi Zheng ${ }^{1}$ and Peng Chen ${ }^{2}$
}

\begin{abstract}
Background: Osteoarthritis $(\mathrm{OA})$ is a degenerative joint disease characterized by articular cartilage degradation and joint inflammation, in which growth factors are significantly involved. The extracellular signal-regulated p38 MAPK pathways play important roles in the regulation of osteogenic and chondrogenic differentiation in bone marrow mesenchymal stem cells (BMSCs). However, the exact mechanism remains unclear.

Methods: In this study, the chondrogenic differentiation of human BMSCs was initiated in micromass culture in the presence of TGF- $\beta 1$ for 14 days. Quantitative RT-PCR and Western blot were performed to detect the transfection effect of shRNA-p38 interfering plasmid in BMSCs. The protein expressions of p/t-p38, SOX9, collagen II, Aggrecan, p/t-Smad1, and p/t-Smad4, as well as the kinase activities of p38/ERK/JNK pathway, were investigated using Western blot analysis. Additionally, the level of chondroitin sulfate and glycosaminoglycans (GAG) expression were measured by Alcian blue staining and GAG assay kit via qualitative and quantitative methods, respectively.

Results: The results demonstrated that p38 pathway was activated in the chondrogenic differentiation of BMSCs induced by TGF- $\beta 1$. Cartilage-specific genes and chondrogenic regulators, such as SOX9, collagen II, Aggrecan, and GAG, were upregulated by TGF- $\beta 1$, which could be reversed by predisposed with shRNA-p38 interfering plasmid and p38-MAPK inhibitors (SB203580). Moreover, the activation of p38/ERK/JNK pathways in the presence of TGF- $\beta 1$ was suppressed by shRNA-p38 and SB203580 treatment.

Conclusion: Collectively, the activation of p38/ERK/JNK/Smad pathways plays a facilitated role in the chondrogenic differentiation induced by TGF- $\beta 1$. After suppressing the p38 pathway, the chondrogenesis can be inhibited, which can be used to guide the treatment of osteoarthritis.
\end{abstract}

Keywords: Osteoarthritis, Chondrogenic differentiation, p38-MAPK, BMSCs, TGF- $\beta 1$

\section{Introduction}

Osteoarthritis (OA) is a chronic joint disorder, which has been the leading cause of disability worldwide, particularly in the elderly suffering from pain and locomotor limitation [1-3]. It was proved to primarily occur in the hip and knee and results from the increase of age, obesity, strain, and injury $[4,5]$. Chondrogenesis is mainly inducted by mesenchymal stem cell (MSC) condensation and differentiation into chondrocytes, which is regarded as the clinical treatment of OA $[6,7]$. In

\footnotetext{
* Correspondence: doctorchen51@163.com

Department of Orthopedics, Yan Cheng Third People's Hospital (Affiliated Yancheng Hospital of Southeast University Medical College), No.2 Xindu West Road, Yancheng 224001, Jiangsu, China

Full list of author information is available at the end of the article
}

addition, bone marrow MSCs possess the ability of multi-differentiation, such as chondrocytes, osteoblasts, and adipocytes, and have been the common seed cells for clinical research and therapy in orthopedics field [8]. However, the chondrogenic differentiation was hardly spontaneous; thus, it is necessary for the researchers to reveal the mechanisms underlying the differentiation from MSCs to chondrocytes, which is an important contribution for cartilage injury repair in OA.

Chondrogenic induction is conducted by forcing aggregation of mesenchymal cells or chondroprogenitor cells to generate a "micromass" or "pellet" culture in a special culture system in vitro [9-11]. TGF- $\beta$ has been widely proven to regulate cell differentiation by setting 
off a large variety of signaling cascades and is tightly controlled by feedback mechanisms at different levels [12]. Mitogen-activated protein kinase (MAPK) family transduction involves a multistep kinase cascade with extracellular signal-regulated protein kinase (ERK), p38 kinase, and c-Jun N-terminal kinase (JNK) [13]. ERK and p38 have been confirmed to occupy core positions in mediating chondrocyte proliferation and related gene expression [14]. Further, MAPKs have been reported as the most important signaling pathway protein kinases, implicated in TGF- $\beta$-mediated chondrocyte functions including cell proliferation, differentiation, apoptosis, and inflammatory responses [15]. However, the definite effects and the potential mechanism of ERK and p38 in chondrogenesis are still unclear.

TGF- $\beta$ triggers a series of downstream pathways via a transmembrane serine/threonine kinase receptor complex, known to be quite complicated. Smads are regarded as central cytoplasmic mediators of TGF- $\beta[16,17]$. Upon TGF- $\beta$ activation, Smad 2 and Smad 3 are activated to form protein complexes with common mediator Smad4, and then phosphorylated and translocated into nucleus to regulate transcription of target genes such as Sox9, Col2, and Aggrecan [18]. Recently, it was reported that p38 and Smad1/5/8 can be simultaneously activated by TGF- $\beta$ activated kinase 1 (TAK1), resulting in the induction of chondrogenic differentiation of stem cells [19]. These findings implied that chondrogenesis initiated by TGF- $\beta 1$ may be tightly regulated through interactions between Smads and p38 MAPK signals. However, the mechanism of p38 pathways in TGF- $\beta$-induced chondrogenesis has not been fully clarified.

In the present study, we employed a micromass chondrogenic culture system for a relatively long duration of 14 days. We sought to investigate the function of $\mathrm{p} 38$ / MEK/ERK/JNK signal pathway in TGF- $\beta$-induced chondrogenesis via regulating cartilage-specific genes for collagen II, Aggrecan, Sox9, and sGAG, and cross-talk between p38 MAPK and Smad1/4 signals in this process.

\section{Materials and methods}

\section{Cell culture and reagent}

Human bone marrow mesenchymal stem cells (BMSCs) were purchased from the American Type Culture Collection and cultured in $\alpha$-modified Eagle medium (Invitrogen, Carlsbad, CA, USA) containing 15\% FBS (Invitrogen), $100 \mathrm{mM}$ L-ascorbic acid 2-phosphate (Wako Pure Chemical Industries, Osaka, Japan), 1\% penicillin and streptomycin (Sigma), and 1\% L-glutamine (Invitrogen). The cells were incubated at $37^{\circ} \mathrm{C}$ in a humidified incubator with $5 \%$ $\mathrm{CO}_{2}$. When hBMSCs were $80-90 \%$ confluent in the culture flasks, the adherent cells were digested with trypsin and subcultured.

\section{Chondrogenesis induction in vitro}

Chondrogenic differentiation of BMSCs was initiated in a micromass culture system. hBMSCs (at passages 3-6) were dissociated for single-cell suspension stating at a density of $2.0 \times 10^{7}$ cells $/ \mathrm{ml}$. Ten-microliter droplets were seeded in culture dishes and allowed to form cell aggregates at $37^{\circ} \mathrm{C}$. In the experimental groups, recombinant human TGF- $\beta 1(10 \mathrm{ng} / \mathrm{ml})$ was added to the chondrogenic medium. Further, micromass cultures were performed for 14 days, and culture media was replaced every 3 days.

\section{Cell transfection and treatment}

Transient transfections of primary chondrocytes were performed as described using Fugene (Roche) [20]. BMSCs were transfected when $60 \%$ of them were fused. The cells were transfected with a shRNA-p38 or an empty expression vector as shRNA-negative control (NC) (Invitrogen), or predisposed with the pharmacological p38 MAPK inhibitors (SB203580, $10 \mu \mathrm{M}$ ) after TGF- $\beta 1$ inducing for 14 days. All the transfections were performed with three independent cell isolations, each of which has quadruplicate replicates.

\section{Real-time quantitative PCR}

Total RNA was isolated from MPC pellets using the RNeasy Mini Kit (Qiagen, Valencia, CA) and quantified spectrophotometrically based on A260. After the first-strand cDNA synthesis using the SuperScript First-Strand Synthesis System (Invitrogen), levels of specific mRNA transcripts were determined by real-time polymerase chain reaction (PCR) utilizing SYBR Green PCR Master Mix and an iCycler realtime PCR detection system (Bio-Rad Laboratories). The primer sequences were as follows: p-p38 forward: $5^{\prime}$-CTGAAC AACATCGTCAAGTGCC-3', reverse: 5' -CATAGCCGGT CATCTCCTCG-3'; p38 forward: 5'-, CCCGAGCGTT ACCAGAACC-3', reverse: 5'- TCGCATGAATGATG GACTGAAAT-3'; GAPDH, forward: 5'-GCACCGTCAA GGCTGAGAAC-3', reverse: 5'-TGGTGAAGACGCCA GTGGA-3'. The $2^{-\Delta \Delta C t}$ method was used in each sample as relative quantification [21]. Each experiment was performed three times.

\section{Western blot analysis}

The BMSCs were collected and lysed at $48 \mathrm{~h}$ after treatment using RIPA buffer. Identical amounts of proteins were resolved by $10-15 \%$ SDS-PAGE and then transferred to a PVDF membrane (Millipore Corporation, Billerica, MA, USA). The membrane was incubated with specific antibodies, Collagen II, Aggrecan, Sox9, p-p38, p38, p-ERK, ERK, p-JNK, JNK, p-Smad1, Smad1, pSmad4, Smad4, and GAPDH (all from Cell Signaling Technology Inc.) at $4{ }^{\circ} \mathrm{C}$ overnight, and peroxidaseconjugated secondary antibodies (KPL, Gaithersburg, 
MD, USA). Chemiluminescence (Millipore Corporation) and densitometry analysis (ImageJ software) were applied to measure the protein expression. Each experiment was performed three times.

\section{Alcian blue staining}

To assess the presence of glycosaminoglycans, which are cartilage-specific matrix proteins, BMSCs were cultured for 14 days in chondrogenic medium and stained with Alcian blue. After removal of the culture medium, the cell-seeded hydrogels were fixed with $4 \%$ paraformalydehyde for $30 \mathrm{~min}$, then washed twice with PBS before the addition of $0.1 \%$ stock solutions of Alcian blue. After 30 min incubation at room temperature, the dye solution was removed and the constructs were washed with distilled water. Moreover, the staining results were recorded under an inverted microscope (Nikon Eclipse TS100). Each experiment was performed three times.

\section{Sulfated glycosaminoglycan quantification}

Colorimetric assessment was performed to determine the amount of the sulfated glycosaminoglycan (sGAG) using a blyscan sulfated glycosaminoglycan assay kit (Biocolor B1000 Std.) according to the manufacturer's instructions. Briefly, $0.25 \mathrm{ml}$ of 1,9-dimethyl-methylene blue (DMMB) dye reagent was added to $80 \mu \mathrm{l}$ of papaindigested extracts and incubated for $30 \mathrm{~min}$ at room temperature. The unbonded dye was removed by centrifugation. Then, the sGAG-bonded dye pellet was dissolved in $0.25 \mathrm{ml}$ of dissociation reagent buffer. A serial dilution of chondroitin 4-sulfate (200 to $1500 \mathrm{ng}$ ) was prepared to generate a standard curve according to the same procedure used for sample preparation. The absorbance was recoded at $525 \mathrm{~nm}$ using a microplate reader (Multiskan Spectrum, Thermo Scientific). Each experiment was performed three times.

\section{Statistical analysis}

Statistical analyses were carried out using Prism 5 software. Each experiment was performed in triplicate and analyzed by one-way ANOVA, followed by Dunnett's post hoc test. Student's $t$ test was used to compare the values of the test and control samples. The results were expressed as mean $\pm \mathrm{SD}$. In all cases, a $p$ value of $<0.05$ was considered to be statistically significant.

\section{Results}

\section{Activation of $\mathrm{p} 38$ in BMSCs after TGF- $\beta 1$ stimulation}

To investigate whether $\mathrm{p} 38$ signal was involved in chondrogenesis induced by TGF- $\beta 1$, the expression of p38 was examined by Western blot analysis. Results showed that the expression of phosphorylated (p)-p38 was exhibited at a low level on day 0 after adding TGF- $\beta 1(10 \mathrm{ng} / \mathrm{ml})$, but gradually increased as chondrogenesis proceeded, and reached at a relatively high level until day 14 compared to day 0 (Fig. 1a, $p<0.05$ at day $5, p<0.01$ at day 7 , and $p<0.001$ at day 14 ). The p-p38 expression in TGF- $\beta$-induced BMSCs was significantly increased in a time-dependent manner. However, the expression of p38 showed no significant difference during 14 days. Therefore, these results indicated that the $\mathrm{p} 38$ signal was activated in chondrogenic differentiation of the BMSCs induced by TGF- $\beta 1$.

As shown in Fig. 1b, at day 0 after adding TGF- $\beta 1$, BMSCs grew adherently to the wall, and the cells were triangular or polygonal in shape. From day 5 to day 14, cell morphology changed significantly and gradually presented a typical paving stone shape with uniform size and shape.

\section{Inhibition of $\mathrm{p} 38$ signals suppressed the chondrogenic differentiation in TGF- $\beta 1$-induced BMSCs}

The overexpression of p-p38 in TGF- $\beta$-induced BMSCs indicated that p38 signal pathway might function as an enhancer of the chondrogenic differentiation. To test this hypothesis, we investigated whether inhibition of p38 affects chondrogenic differentiation in TGF- $\beta$-induced BMSCs. In Fig. $2 \mathrm{a}$ and $\mathrm{b}$, the results showed that the protein level of pp38 and the mRNA of p38 were significantly decreased using Western blot and RT-qPCR after being transfected with p38 interfering plasmid (shRNA-p38) in TGF- $\beta$ induced BMSCs $(p<0.001)$. Interestingly, the protein expression of p38 was almost unchanged (Fig. 2a), while its mRNA level decreased significantly in the shRNA-p38 group when compared with control and shRNA-NC groups (Fig. 2b, $p<0.05$ ). Chondrogenic potential of BMSCs was confirmed by examining the expression of cartilage-specific gene coding for collagen II, Aggrecan, Sox9, p/t-smad1, and p/t-smad4 proteins with Western blot. TGF- $\beta$-treated BMSCs exhibited higher expression in all these cartilagespecific genes after 14 days compared with the untreated control group (Fig. 2c, $p<0.001$ ). Treatment of shRNA-p38 and pretreatment of SB203580, TGF- $\beta 1$-induced gene expression of collagen II, Aggrecan, Sox $9, \mathrm{p} / \mathrm{t}$-smad1, and $\mathrm{p} / \mathrm{t}$ smad4 were notably decreased compared with the TGF- $\beta 1$ group (Fig. 2c). Further, the reverse effects of transfection interference plasmids shRNA-p38 were better than that of p38 inhibitors SB203580. Thus, these results indicated that cartilage-specific gene expression was affected by p38 inhibitors in reverse patterns, confirming that $\mathrm{p} 38$ could regulate the process of chondrogenic differentiation.

In addition, a quantitative method was utilized to measure the amount of sulfated glycosaminoglycan (sGAG) deposited in the extracellular matrix (ECM) of the micromasses. The results showed a significant increase with TGF- $\beta 1$ group in sGAG content compared to the control group (Fig. $3 \mathrm{a}, p<0.001$ ), while TGF- $\beta 1$ in combination with shRNA-p38 or SB203580 exhibited a significant decrease in sGAG content compared 
A

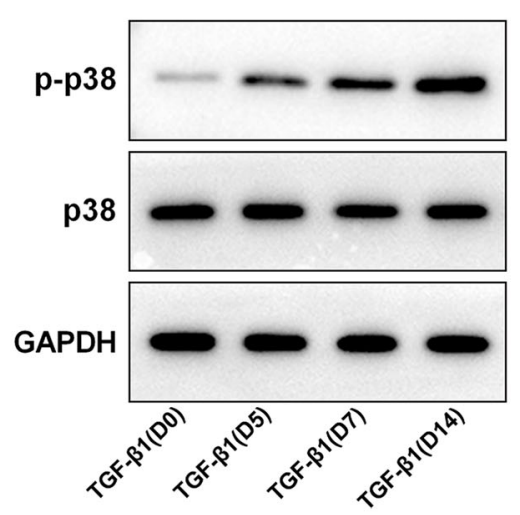

B

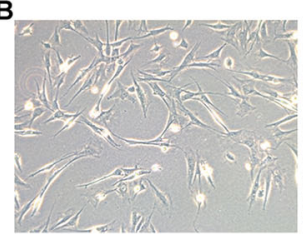

TGF- $\beta 1(D 0)$

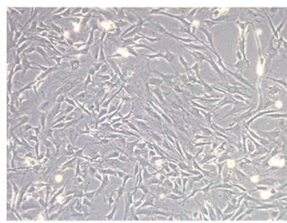

TGF- $\beta 1$ (D5)

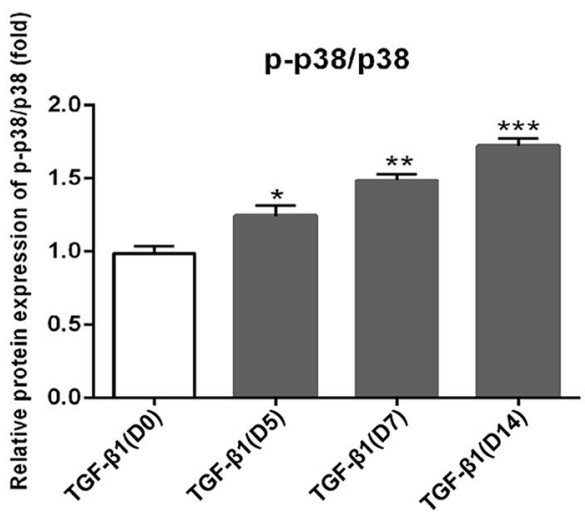

Fig. 1 Expression of p-p38/p38 in TGF- $\beta 1$-induced BMSCs and morphological observation. a Western blotting of p-p38 and p38 expression in BMSCs following TGF- $\beta 1$-induced for $0,5,7$, and 14 days. Relative protein expression of p-p38/p38 was elevated on a time-dependent manner under the TGF- $\beta 1$ induction when compared with day 0 . b Representative images of BMSCs. Morphological changes of BMSCs following TGF- $\beta 1$ induced for 0, 5, 7, and 14 days were observed under an inverted microscope. On day 0, BMSCs grew adherently to the wall, and the cells were triangular or polygonal in shape. From day 5 to day 14, cell morphology changed significantly and gradually presented a typical paving stone shape with uniform size and shape. ${ }^{*} p<0.05,{ }^{* *} p<0.01$, and ${ }^{* *} p<0.001$ vs. TGF- $\beta 1$ (D0)

to the TGF- $\beta 1$ group at day 14 (Fig. 3a, $p<0.01$ ). Therefore, GAG content was significantly regulated by the p38 pathway in TGF- $\beta 1$-induced BMSCs. According to the results of Alcian blue staining, the chondrogenic differentiation (central blue nucleus) of BMSCs was significantly enhanced in the TGF- $\beta 1$ group, while suppression of $\mathrm{p} 38$ could inhibit the chondrogenic differentiation of BMSCs (Fig. 3b).

\section{Inhibition of p38 signals suppressed the p38/ERK/JNK pathway in the presence of TGF- $\beta 1$-induced chondrogenesis}

The activation of p38/ERK/JNK was assessed using Western blot after treating with p38 inhibitors in TGF$\beta 1$-induced BMSCs. The results in Fig. 4 indicated that the expressions of p-p38, p-ERK, and p-JNK were overexpressed compared with the control group $(p<0.001)$. Both shRNA-p38 and SB203580 could decrease the expression of p-p38, p-ERK, and p-JNK in TGF- $\beta 1$ induced BMSCs. Therefore, these results showed that p38 may promote chondrogenic differentiation by regulating $\mathrm{p} 38 / \mathrm{ERK} / \mathrm{JNK}$ signal pathways.

\section{Discussion}

Adult human MSCs are considered as promising candidate cell sources for use in cartilage tissue engineering applications. In this study, we further clarify the involvement of intracellular signaling in the regulation of BMSC cartilage formation. The TGF- $\beta 1$-induced micromass culture system was employed in order to analyze the roles of p38 in the chondrogenic differentiation of BMSCs. This study demonstrated that p38/ ERK/JNK pathway was activated by TGF- $\beta 1$ in chondrogenesis of BMSCs, and played crucial roles in gene transcription of cartilage-specific markers coding for Aggrecan, SOX9, collagen II, Smad1/4, and sGAG. The study also provided evidences that p38 might regulate TGF- $\beta 1$-induced chondrogenesis and directly interact with $\mathrm{p} 38 / \mathrm{ERK} / \mathrm{JNK}$ pathway.

In terms of osteogenic differentiation, previous studies indicated that p38 and ERK were involved in bone morphogenetic factor-9-induced osteogenic differentiation of rat dental follicle stem cells [22]. Particularly, p38/MAPK was accepted as a positive regulator in TGF- $\beta$-induced BMSCs [23]. In the present study, p38 signal was activated in chondrogenic differentiation of the BMSCs induced by TGF- $\beta$ after 14 days. The results of Western blot revealed that both shRNA-p38 and SB203580 could decrease the p-p38 expression in TGF- $\beta$-induced BMSCs. These results were consistent with previous conclusions. However, the function of the ERK signal remains complex and inconclusive. Prior study reported that cyclic tensile stress may enhance the osteogenic differentiation of periodontal ligament cells via the ERK signaling pathway, and the inhibition of ERK may 


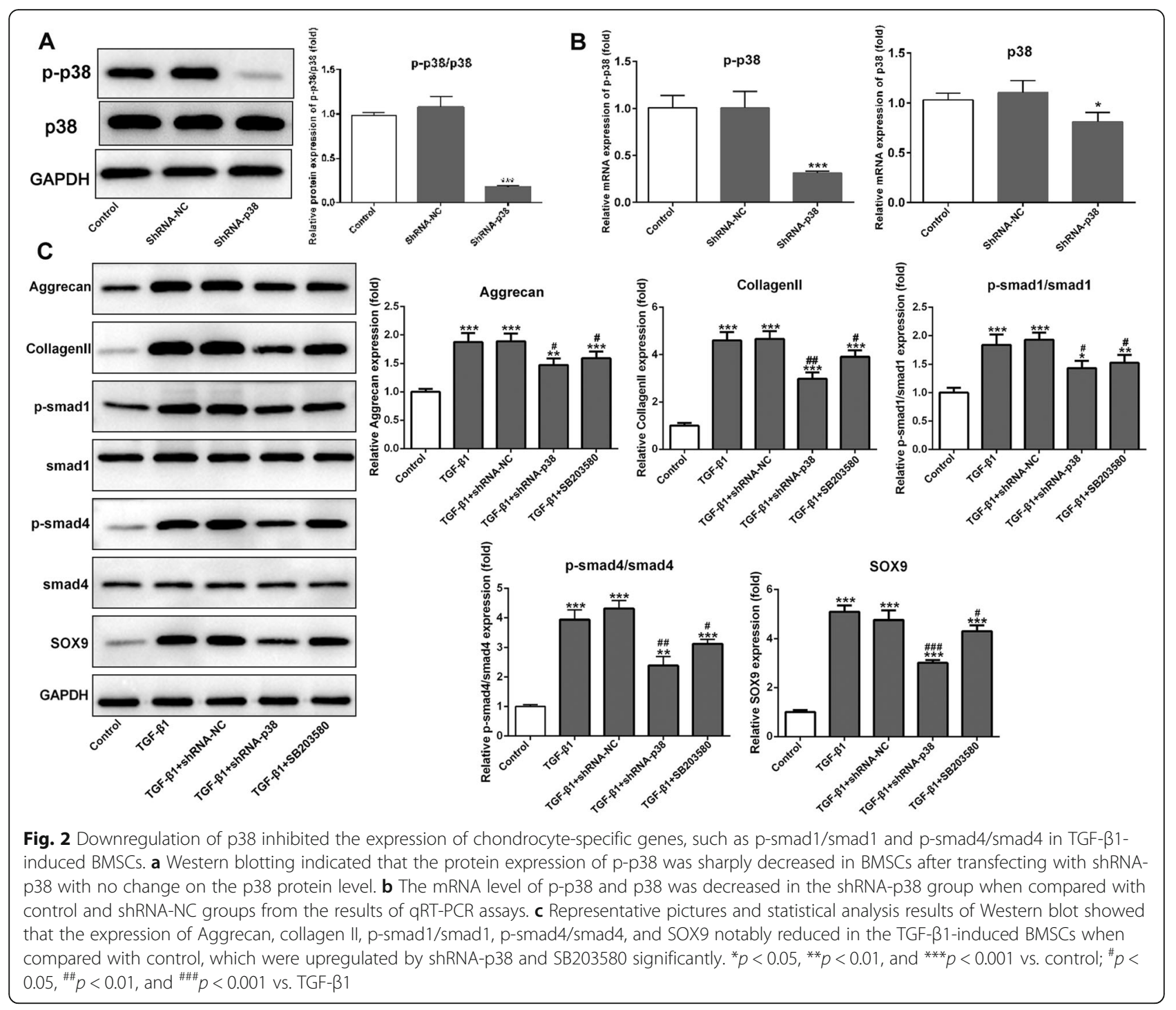

inhibit osteogenic differentiation [24]. In another study, inhibition of p38 may suppress the osteogenic differentiation of dental pulp stem cells (DPSCs), whereas inhibition of ERK demonstrated the opposite effect [25]. In general, differences between cell type, chondrogenic culture systems, chondrogenic developmental stages, and inhibitor concentrations may be the reason that leads to variations between these results, and diverse methods for detection of chondrocyte differentiation were also regarded as critical factors to explain conflicting results due to differences in accuracy and efficiency [26]. In this study, hBMSCs were derived from $\mathrm{BM}$ of healthy adults, and a micromass chondrogenic culture system was employed. The methods have been validated by previous studies in micromass cultures of chick limb bud mesenchyme, pellet cultures of adult MPCs, and mouse chondrogenic ATDC5 cells [27-29]. Prior study revealed that phosphorylation of p38 was relatively stable and prolonged in chondrogenesis stimulated by TGF- $\beta 1$, but the
ERK subtype was phosphorylated in a rapid and transient manner [26]. In our study, the hMSC detection was optimized for a relatively appropriate duration of 14 days. It was found that the expressions of p-ERK, p-p38, and p-JNK were overexpressed in TGF- $\beta 1$-induced hBMSCs, which could be reversed by p38 inhibitors. This may explain why ERK phosphorylation was relatively difficult to detect.

Chondrogenesis progresses with the expression of a cascade of stage-specific markers. Sox 9 protein has been proven to be a master regulatory factor for chondrocyte differentiation [30]. It can transcriptionally activate cartilage-specific marker gene coding for collagen II and regulate synthesis of Aggrecan [31]. In our study, TGF$\beta 1$-treated cultures exhibited higher expression in collagen II, Aggrecan, and SOX9 after 14 days compared with the untreated controls, which were decreased by pretreating p38 inhibitors. Moreover, TGF- $\beta 1$ induced the chondrogenic differentiation of MSCs both in monolayer 


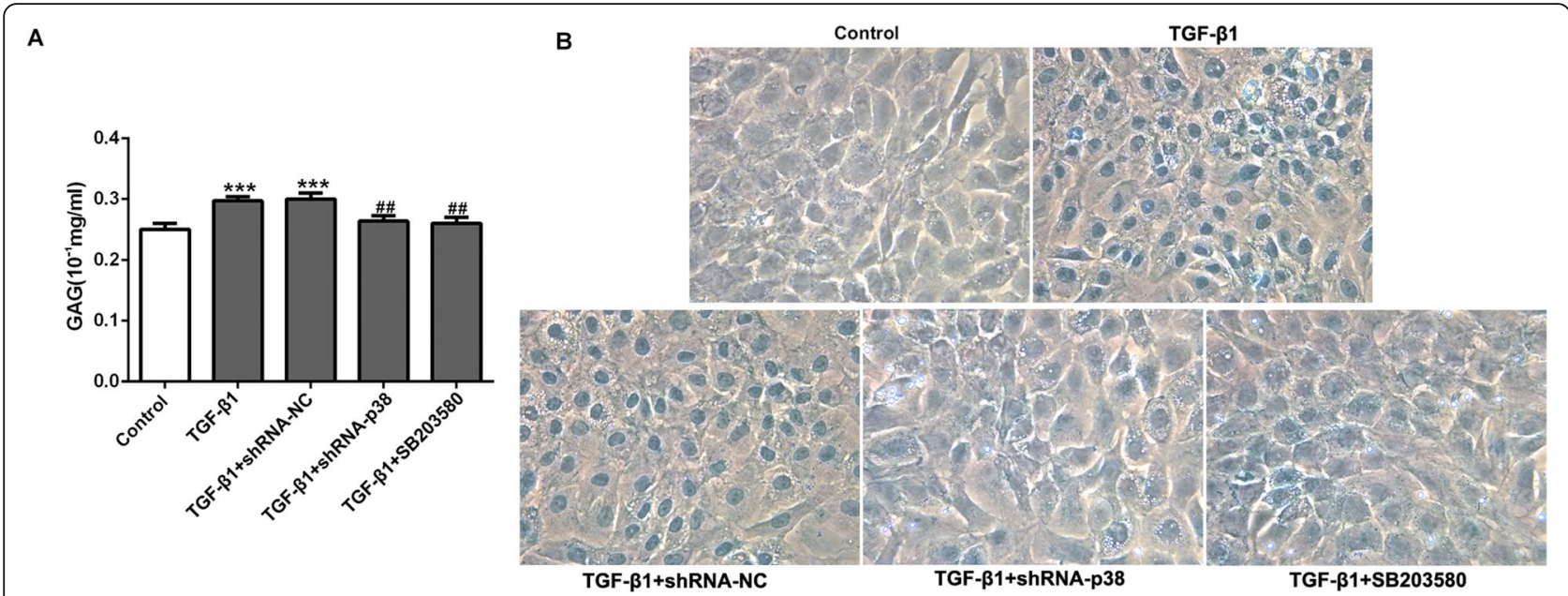

Fig. 3 Effects of p38 inhibition on GAG level and the degree of chondrocyte differentiation in TGF- $\beta 1$-induced BMSCs. a GAG assay and $\mathbf{b}$ Alcian blue staining showed that the GAG level and the number of blue-stained cells (the nuclei of chondrocytes can be blue-stained) in BMSCs were distinctly increased in TGF- $\beta 1$-induced BMSCS, while p38 silence inhibited chondrogenic differentiation significantly. ${ }^{* * *} p<0.001$ vs. control; ${ }^{\# \#} p<$ 0.01 vs. TGF- $\beta 1$

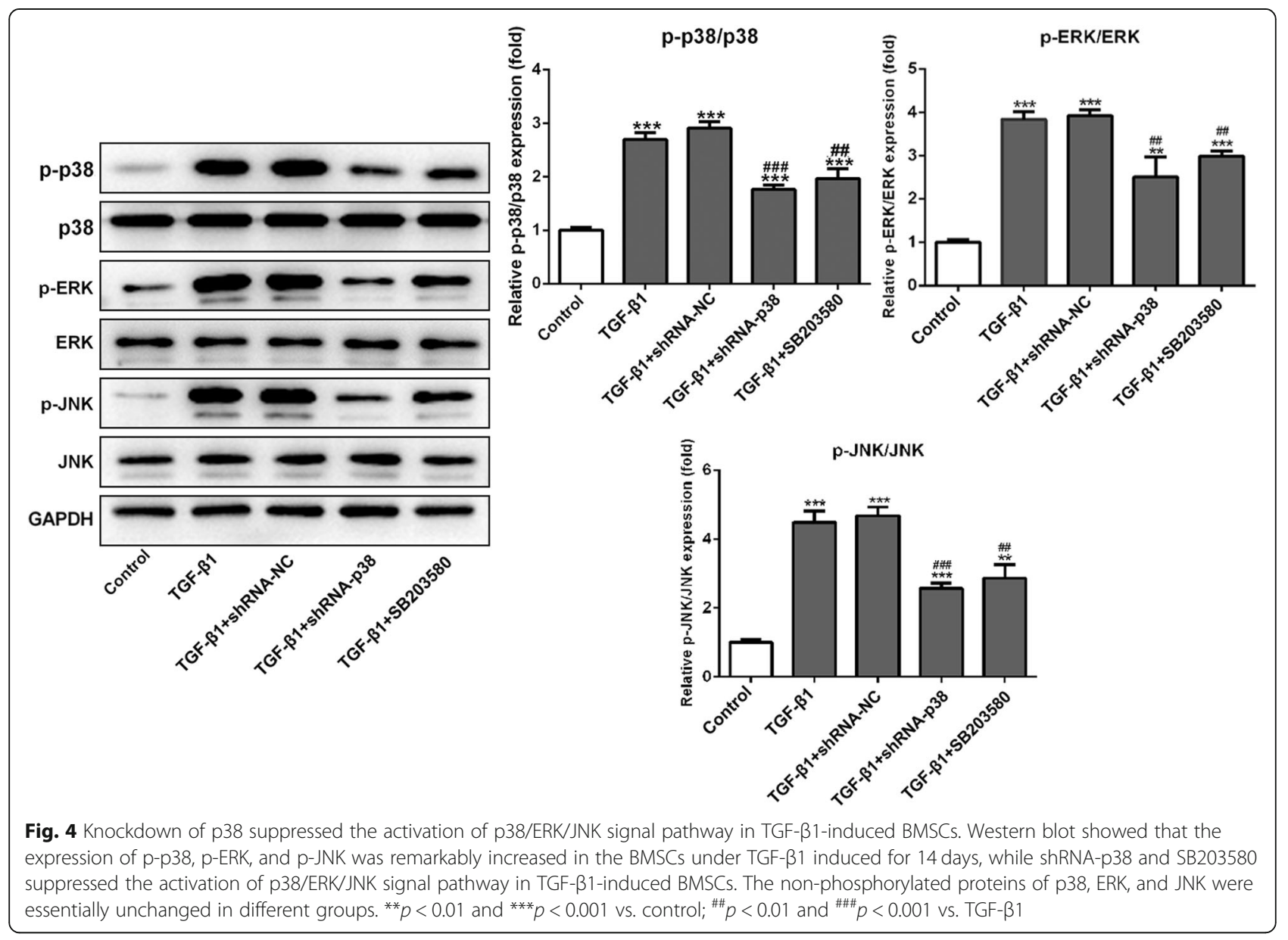


and in the collagen-GAG scaffold. In previous study, the treatment of MSC-seeded collagen-GAG scaffolds with TGF- $\beta$ resulted in an increase in $\left[{ }^{35} \mathrm{~S}\right]$ sulfate incorporation which was reduced upon inhibition of the p38 pathway [18]. In our study, inhibition of the p38 led to a significant reduction of sGAG synthesis by Alcian blue staining and sGAG assay detection, which were consistent with the previous studies. Therefore, our study indicated that TGF- $\beta 1$-treated BMSCs differentiated into chondrocytes with expression of chondrocyte-specific genes, and the p38 pathway delicately regulated chondrogenesis induced by TGF- $\beta 1$.

In previous study, only gene expression of $\mathrm{Smad} 2 / 3$ was examined, and further studies of Smad proteins should be investigated for the interaction between MAPK and Smad signaling on TGF- $\beta$ stimulation [32]. Interactions between p38 pathways and Smad1/4 were investigated in the process of chondrogenesis stimulated by TGF- $\beta 1$. TGF- $\beta$-induced Smad1/4 overexpression was reduced by inhibition of p38 with shRNA-p38 or SB203580, leading to the downregulation of chondrocyte-specific genes such as collagen II and Aggrecan. Therefore, our findings confirmed that p38 MAPK signal might regulate chondrocyte-specific genes by mediating interaction between TGF- $\beta 1$ and Smad1/4 molecules. This may provide a new perspective on the control of TGF$\beta$-dependent transcriptional activation in the process of chondrogenesis.

\section{Conclusion}

In conclusion, the present study proved that $\mathrm{p} 38$ signals were activated in TGF- $\beta$-induced BMSCs, and inhibition of p38 regulated the chondrogenic-specific genes such as SOX9, collagen II, Aggrecan, and sGAG level, through p38/ERK/JNK pathway in TGF- $\beta$-induced BMSCs. Meanwhile, it was observed that cross-talk mechanisms between p38 and Smad1/4 pathways were involved in transcriptional regulation of chondrocyte-specific genes stimulated by TGF- $\beta 1$. These results will deepen our understanding of the mechanism of chondrogenesis process, which provided clues for the application of multipotential stem cells and the treatment of osteoarthritis.

\section{Abbreviations}

BMSCs: Bone marrow mesenchymal stem cells; ERK: Extracellular signalregulated protein kinase; GAG: Glycosaminoglycans; JNK: C-Jun N-terminal kinase; OA: Osteoarthritis; TAK1: TGF- $\beta$-activated kinase 1

\section{Acknowledgements}

Not applicable.

\section{Authors' contributions}

$\mathrm{NM}, \mathrm{XT}$, and $\mathrm{PC}$ analyzed the data and wrote the manuscript. $\mathrm{NM}, \mathrm{XT}$, and QZ performed the experiments. NM and PC created the study concept and designed the experiments. All authors read and approved the final manuscript.

\section{Funding}

Not applicable.

\section{Availability of data and materials}

The datasets used and/or analyzed during the current study are available from the corresponding author on reasonable request.

Ethics approval and consent to participate

Not applicable.

\section{Consent for publication}

Not applicable.

\section{Competing interests}

The authors declare that they have no competing interests.

\section{Author details}

'Department of Orthopedics, Zhejiang Taizhou Central Hospital (Affiliated Hospital of Taizhou University), No. 999 Donghai Avenue, Jiaojiang District, Taizhou 318000, Zhejiang, China. ²Department of Orthopedics, Yan Cheng Third People's Hospital (Affiliated Yancheng Hospital of Southeast University Medical College), No.2 Xindu West Road, Yancheng 224001, Jiangsu, China.

Received: 15 August 2019 Accepted: 28 November 2019

Published online: 12 December 2019

References

1. Felson DT, Lawrence RC, Dieppe PA, Hirsch R, Helmick CG, Jordan JM, Kington RS, Lane NE, Nevitt MC, Zhang Y, Sowers M, McAlindon T, Spector TD, Poole AR, Yanovski SZ, Ateshian G, Sharma L, Buckwalter JA, Brandt KD, Fries JF. Osteoarthritis: new insights. Part 1: the disease and its risk factors. Ann Intern Med. 2000;133:635-46.

2. McCulloch K, Litherland GJ, Rai TS. Cellular senescence in osteoarthritis pathology. Aging Cell. 2017;16:210-8.

3. Bortoluzzi A, Furini F, Scire CA. Osteoarthritis and its management - epidemiology, nutritional aspects and environmental factors. Autoimmun Rev. 2018:17:1097-104.

4. Woolf AD, Pfleger B. Burden of major musculoskeletal conditions. Bull World Health Organ. 2003;81:646-56.

5. Green JA, Hirst-Jones KL, Davidson RK, Jupp O, Bao Y, MacGregor AJ, Donell ST, Cassidy A, Clark IM. The potential for dietary factors to prevent or treat osteoarthritis. Proc Nutr Soc. 2014;73:278-88.

6. Tang C, Jin C, Xu Y, Wei B, Wang L. Chondrogenic differentiation could be induced by autologous bone marrow mesenchymal stem cell-derived extracellular matrix scaffolds without exogenous growth factor. Tissue Eng Part A. 2016;22:222-32.

7. Li P, Gong Z, Shultz LD, Ren G. Mesenchymal stem cells: from regeneration to cancer. Pharmacol Ther. 2019;200:42-54.

8. Fu X, Liu G, Halim A, Ju Y, Luo Q, Song AG. Mesenchymal stem cell migration and tissue repair. Cells. 2019;8(8):784.

9. Tuli R, Tuli S, Nandi S, Huang X, Manner PA, Hozack WJ, Danielson KG, Hall DJ, Tuan RS. Transforming growth factor-beta-mediated chondrogenesis of human mesenchymal progenitor cells involves $\mathrm{N}$-cadherin and mitogenactivated protein kinase and Wnt signaling cross-talk. J Biol Chem. 2003;278: 41227-36.

10. Spitkovsky D, Hescheler J. Adult mesenchymal stromal stem cells for therapeutic applications. Minim Invasive Ther Allied Technol. 2008;17:79-90.

11. Sierra-Sanchez A, Ordonez-Luque A, Espinosa-Ibanez O, Ruiz-Garcia A, AriasSantiago S. Epithelial in vitro differentiation of mesenchymal stem cells. Curr Stem Cell Res Ther. 2018;13:409-22.

12. Ihara S, Hirata Y, Koike K. TGF-beta in inflammatory bowel disease: a key regulator of immune cells, epithelium, and the intestinal microbiota. J Gastroenterol. 2017:52:777-87.

13. Zhang M, Su J, Zhang Y, Xu J, Zhang S. Conveying endogenous and exogenous signals: MAPK cascades in plant growth and defense. Curr Opin Plant Biol. 2018:45:1-10.

14. Krens SF, Spaink HP, Snaar-Jagalska BE. Functions of the MAPK family in vertebrate-development. FEBS Lett. 2006:580:4984-90.

15. Chen G, Deng C, Li YP. TGF-beta and BMP signaling in osteoblast differentiation and bone formation. Int J Biol Sci. 2012:8:272-88.

16. Lan HY, Chung ACK. Transforming growth factor-beta and Smads. Contrib Nephrol. 2011;170:75-82

17. Ooshima A, Park J, Kim SJ. Phosphorylation status at Smad3 linker region modulates transforming growth factor-beta-induced epithelialmesenchymal transition and cancer progression. Cancer Sci. 2019;1 10:481-8. 
18. McMahon LA, Prendergast PJ, Campbell VA. A comparison of the involvement of $\mathrm{p} 38, \mathrm{ERK} 1 / 2$ and PI3K in growth factor-induced chondrogenic differentiation of mesenchymal stem cells. Biochem Biophys Res Commun. 2008;368:990-5.

19. Blaney Davidson EN, van Caam AP, Vitters EL, Bennink MB, Thijssen E, van den Berg WB, Koenders Ml, van Lent PL, van de Loo FA, van der Kraan PM. TGF-beta is a potent inducer of nerve growth factor in articular cartilage via the ALK5-Smad2/3 pathway. Potential role in OA related pain? Osteoarthr Cartil. 2015;23:478-86.

20. Woods A, Beier F. RhoA/ROCK signaling regulates chondrogenesis in a context-dependent manner. J Biol Chem. 2006;281:13134-40.

21. Livak KJ, Schmittgen TD. Analysis of relative gene expression data using real-time quantitative PCR and the 2(-Delta Delta C(T)) method. Methods. 2001;25:402-8.

22. Li C, Yang X, He Y, Ye G, Li X, Zhang X, Zhou L, Deng F. Bone morphogenetic protein-9 induces osteogenic differentiation of rat dental follicle stem cells in P38 and ERK1/2 MAPK dependent manner. Int J Med Sci. 2012;9:862-71.

23. Oh CD, Chang SH, Yoon YM, Lee SJ, Lee YS, Kang SS, Chun JS. Opposing role of mitogen-activated protein kinase subtypes, erk-1/2 and p38, in the regulation of chondrogenesis of mesenchymes. J Biol Chem. 2000;275: 5613-9.

24. Li L, Han M, Li S, Wang L, Xu Y. Cyclic tensile stress during physiological occlusal force enhances osteogenic differentiation of human periodontal ligament cells via ERK1/2-Elk1 MAPK pathway. DNA Cell Biol. 2013;32:488-97.

25. Ba P, Duan X, Fu G, Lv S, Yang P, Sun Q. Differential effects of p38 and Erk1/ 2 on the chondrogenic and osteogenic differentiation of dental pulp stem cells. Mol Med Rep. 2017;16:63-8.

26. Li J, Zhao Z, Liu J, Huang N, Long D, Wang J, Li X, Liu Y. MEK/ERK and p38 MAPK regulate chondrogenesis of rat bone marrow mesenchymal stem cells through delicate interaction with TGF-beta1/Smads pathway. Cell Prolif. 2010;43:333-43.

27. Chang SH, Oh CD, Yang MS, Kang SS, Lee YS, Sonn JK, Chun JS. Protein kinase $C$ regulates chondrogenesis of mesenchymes via mitogen-activated protein kinase signaling. J Biol Chem. 1998;273:19213-9.

28. Li J, Wang J, Zou Y, Zhang Y, Long D, Lei L, Tan L, Ye R, Wang X, Zhao Z. The influence of delayed compressive stress on TGF-betal-induced chondrogenic differentiation of rat BMSCs through Smad-dependent and Smad-independent pathways. Biomaterials. 2012;33:8395-405.

29. Goto K, Kimura T, Kitamura N, Semba S, Ohmiya Y, Aburatani S, Matsukura S, Tsuda M, Kurokawa T, Ping Gong J, Tanaka S, Yasuda K. Synthetic PAMPS gel activates BMP/Smad signaling pathway in ATDC5 cells, which plays a significant role in the gel-induced chondrogenic differentiation. J Biomed Mater Res A. 2016:104:734-46.

30. Jiang $X$, Huang $X$, Jiang $T$, Zheng $L$, Zhao J, Zhang $X$. The role of Sox9 in collagen hydrogel-mediated chondrogenic differentiation of adult mesenchymal stem cells (MSCs). Biomater Sci. 2018;6:1556-68.

31. Xie WF, Zhang X, Sakano S, Lefebvre V, Sandell L. Trans-activation of the mouse cartilage-derived retinoic acid-sensitive protein gene by Sox9. J Bone Miner Res. 1999;14:757-63.

32. Madej W, Buma P, van der Kraan P. Inflammatory conditions partly impair the mechanically mediated activation of Smad2/3 signaling in articular cartilage. Arthritis Res Ther. 2016;18:146.

\section{Publisher's Note}

Springer Nature remains neutral with regard to jurisdictional claims in published maps and institutional affiliations.

Ready to submit your research? Choose BMC and benefit from:
- fast, convenient online submission
- thorough peer review by experienced researchers in your field
- rapid publication on acceptance
- support for research data, including large and complex data types
- gold Open Access which fosters wider collaboration and increased citations
- maximum visibility for your research: over 100M website views per year
At BMC, research is always in progress.
Learn more biomedcentral.com/submissions

